\title{
Unilateral prefrontal lobotomy for epilepsy: technique and surgical anatomy
}

\author{
Giulia Cossu, MD, ${ }^{1}$ Pablo González-López, MD, PhD, ${ }^{2}$ Etienne Pralong, MD, ${ }^{1}$ Judith Kalser, MD, ${ }^{3}$ \\ Mahmoud Messerer, MD, MSc, ${ }^{1}$ and Roy Thomas Daniel, MD, MCh' \\ 'Department of Neurosurgery, University Hospital of Lausanne; ${ }^{2}$ Department of Pediatrics, Section of Neuro-Pediatrics, \\ University Hospital of Lausanne, Switzerland; and '2Department of Neurosurgery, Hospital General Universitario de Alicante, \\ Spain
}

OBJECTIVE Surgery for frontal lobe epilepsy remains a challenge because of the variable seizure outcomes after surgery. Disconnective procedures are increasingly applied to isolate the epileptogenic focus and avoid complications related to extensive brain resection. Previously, the authors described the anterior quadrant disconnection procedure to treat large frontal lobe lesions extending up to but not involving the primary motor cortex. In this article, they describe a surgical technique for unilateral disconnection of the prefrontal cortex, while providing an accurate description of the surgical and functional anatomy of this disconnective procedure.

METHODS The authors report the surgical treatment of a 5-month-old boy who presented with refractory epilepsy due to extensive cortical dysplasia of the left prefrontal lobe. In addition, with the aim of both describing the subcortical intrinsic anatomy and illustrating the different connections between the prefrontal lobe and the rest of the brain, the authors dissected six human cadaveric brain hemispheres. These dissections were performed from lateral to medial and from medial to lateral to reveal the various tracts sectioned during the three different steps in the surgery, namely the intrafrontal disconnection, anterior callosotomy, and frontobasal disconnection.

RESULTS The first step of the dissection involves cutting the $U$-fibers. During the anterior intrafrontal disconnection, the superior longitudinal fasciculus in the depth of the middle frontal gyrus, the uncinate fasciculus, and the inferior frontooccipital fasciculus in the depth of the inferior frontal gyrus at the level of the anterior insular point are visualized and sectioned, followed by sectioning of the anterior limb of the internal capsule. Once the frontal horn is reached, the anterior callosotomy can be performed to disconnect the genu and the rostrum of the corpus callosum. The intrafrontal disconnection is deepened toward the falx, and at the medial surface, the cingulum is sectioned. The frontobasal disconnection involves cutting the anterior limb of the anterior commissure.

CONCLUSIONS This technique allows selective isolation of the epileptogenic focus located in the prefrontal lobe to avoid secondary propagation. Understanding the surface and white matter fiber anatomy is essential to safely perform the procedure and obtain a favorable seizure outcome.

https://thejns.org/doi/abs/10.3171/2020.1.FOCUS19938

KEYWORDS epilepsy surgery; disconnection; prefrontal lobe; cortical dysplasia

$\mathrm{F}$ RONTAL lobe epilepsy (FLE) is the second most common form of focal epilepsy ${ }^{39}$ and accounts for about $20 \%$ of patients with refractory epilepsy. $5,16,17,35$ The surgical outcomes are less than satisfying compared to those in temporal lobe epilepsy. And while long-term success rates vary from $20 \%$ to $80 \%,{ }^{22,43}$ most studies report an Engel class I seizure outcome in about $50 \%$ of patients with FLE. $18,21,24,26,27,36,38,44,45$ Definition of the epileptogenic zone is the mainstay of surgical treatment and can be ascertained by a concordance of data from seizure semiology, electroencephalography, FDG-PET, SPECT, and MRI. Resective surgery of the epileptogenic lesion aims at removal of the entire epileptogenic zone without inducing a new neurological deficit. Disconnection of the epileptogenic zone is an attractive alternative with the same objective. In general, disconnective techniques are favored for large epileptogenic lesions with a view to avoiding cavity complications related to extensive brain excision. ${ }^{12,15,40,42}$ 

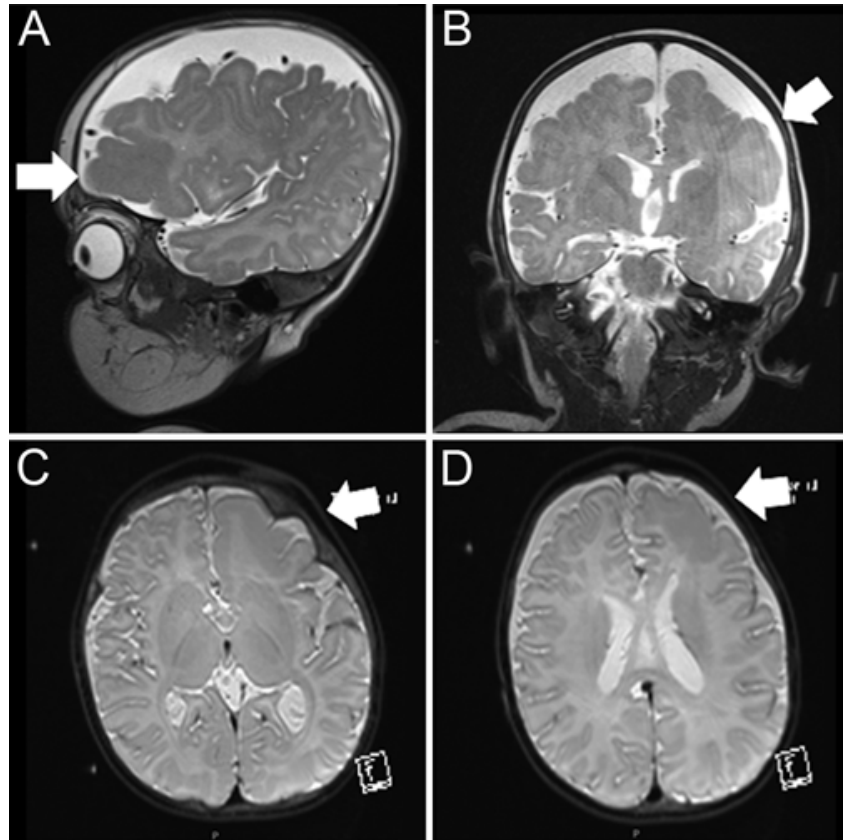

FIG. 1. Preoperative cerebral T2-weighted MRI showing dysplasia in the left prefrontal lobe. In the sagittal plane (A) dysplasia involved the cortex anterior to the triangular gyrus (arrow), while in the coronal (B) and axial ( $C$ and $\mathbf{D}$ ) planes involvement of the medial cortex (arrows) can be appreciated. Electrophysiological studies confirmed the location of the epileptogenic focus in this area.

FLE semiology is diverse, and efforts at sublobar localization and classification are challenging. FLE can be classified as dorsolateral, mesial, or basal ${ }^{3}$ to allow selective resection or disconnection of the epileptogenic focus while avoiding damage to healthy and eloquent areas. Dorsolateral FLE can be further divided into central, premotor, and prefrontal.

We previously described the periinsular anterior quadrantotomy technique to treat extensive dysplasia involving the frontal lobe anterior to the motor cortex and thus involving the prefrontal and premotor cortices. ${ }^{6}$ The aim of the present paper was to describe a similar disconnection technique safely performed to disconnect the entire prefrontal cortex (sparing the motor and premotor areas) in a child presenting with focal cortical dysplasia and refractory epilepsy localized to the left prefrontal lobe. In addition, with the aim of both describing the subcortical intrinsic anatomy and illustrating the different connections between the prefrontal lobe and the rest of the brain, we dissected six human cadaveric brain hemispheres. These dissections were performed from lateral to medial and from medial to lateral to reveal the various tracts sectioned during the three different steps in the surgery, namely the intrafrontal disconnection, anterior callosotomy, and frontobasal disconnection.

\section{Illustrative Case}

\section{History and Examination}

A 3-month-old infant presented with refractory focal epilepsy. He had been born prematurely at 28 weeks' ges-
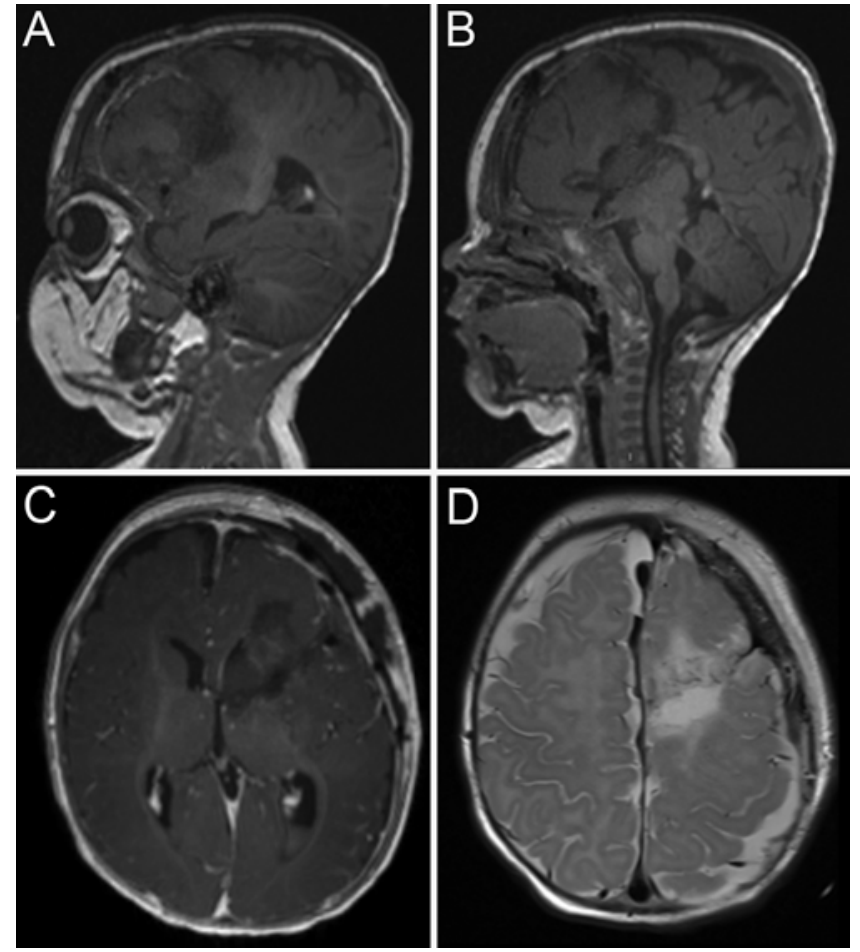

FIG. 2. Postoperative sagittal T1-weighted (A and B) and axial T1weighted (C) and T2-weighted (D) MRI illustrating the intrafrontal disconnection. The results of the anterior callosotomy with disconnection of the genu and the rostrum are visible in panel B. The prefrontal cortex was completely disconnected.

tation and presented with frequent daily seizures manifesting as leftward conjugate eye deviation and bilateral clonic movements of the eyelids, followed by clonic movements of the right-sided limbs, followed by clonic movements of all four limbs. Seizures were refractory to four antiepileptic drugs (AEDs). Interictal EEG showed a left prefrontal epileptic focus. Numerous seizures were recorded on EEG, always starting in the left frontal areas and rapidly spreading posteriorly and to the contralateral hemisphere. Cerebral MRI confirmed the presence of cortical dysplasia involving the entire prefrontal lobe including the mesial and basal parts of the lobe (Fig. 1). These areas were hypometabolic on interictal PET scan. After a multidisciplinary discussion and considering the refractory nature of the disease and the excellent concordance with phase 1 evaluations (semiology and electrophysiological and radiological studies), a unilateral prefrontal lobotomy was proposed to disconnect the epileptogenic focus in its entirety. Because of the infant's prematurity and low birth weight, however, we waited 2 months till he gained weight in order to reduce the anesthesia risk at surgery.

\section{Operative and Postoperative Courses}

The child underwent surgery at the age of 5 months (corrected age 2.5 months) with a weight of $5 \mathrm{~kg}$. In the early postoperative period, he presented with transient conjugate nystagmus to the right side and mild paresis of the right upper limb, from which he completely recovered. 

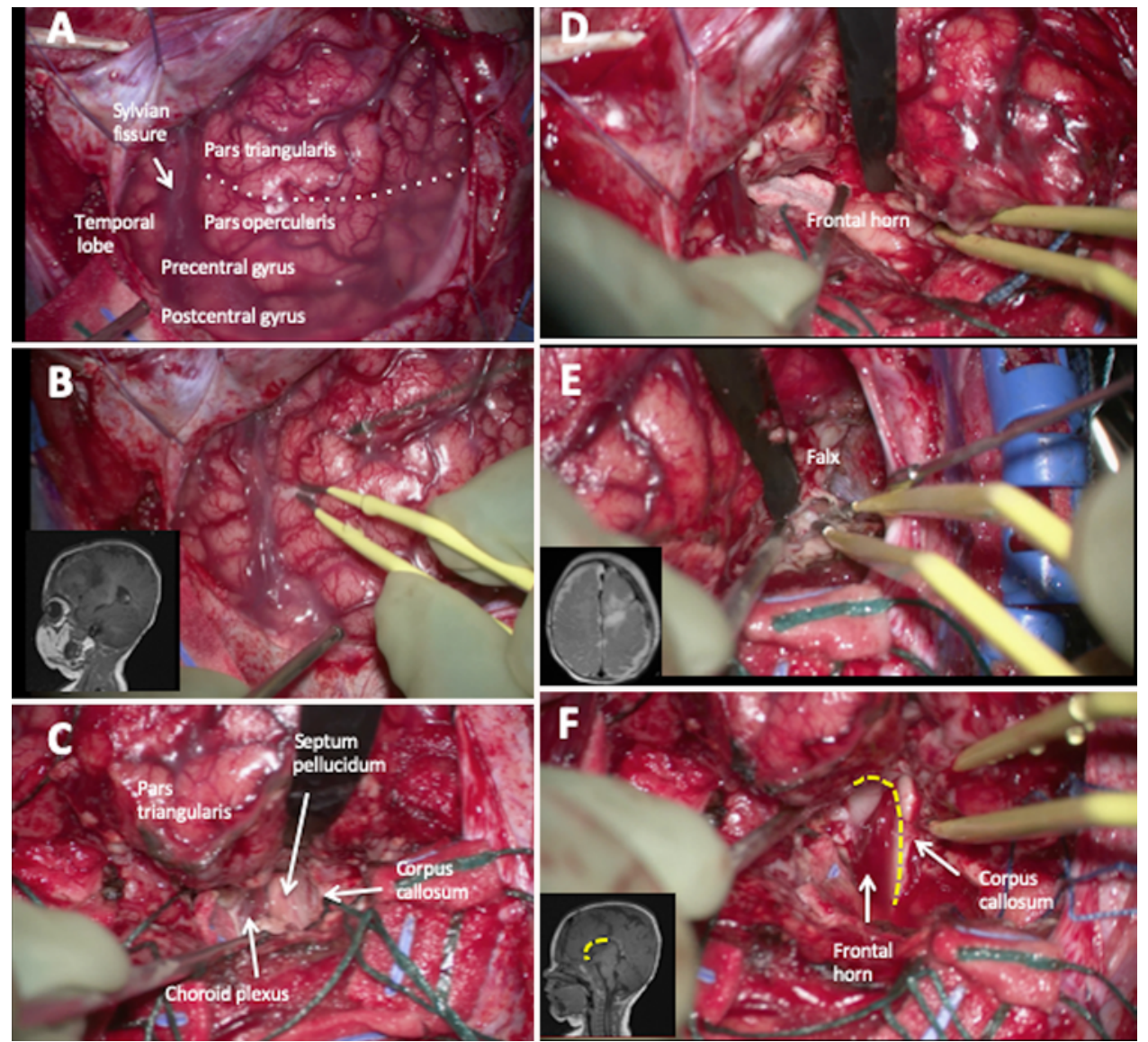

FIG. 3. Once the electrophysiological study is performed and the pars triangularis as well as the pars opercularis are identified (A), a corticotomy between these two portions of the inferior frontal gyrus is performed (B) going toward the vertex. White dotted line indicates the cortical incision between the pars triangularis and pars opercularis. In the depth, once the external capsule and the anterior limb of the internal capsule are opened, the frontal horn of the lateral ventricle is reached (C and D). Superiorly, the disconnection is deepened till the falx is reached in order to also disconnect the mesial part of the lobe $(E)$. From the ventricle, the anterior callosotomy is performed to disconnect the genu and the rostrum of the corpus callosum (F). Yellow dashed line denotes the disconnection of the anterior part of the corpus callosum.

At the latest follow-up 1 year after surgery, there were no focal neurological deficits. The patient had two episodes of rapid eye movements with retained awareness in the early postoperative period. No other clinical seizures were recorded. He was considered to have an Engel class II seizure outcome at the last follow-up. Three of the four AEDs have been tapered off to date. Postoperative MRI confirmed disconnection of the left prefrontal lobe (Fig. 2), and EEG performed 3 months after surgery showed isolated epileptiform activity from the disconnected lobe with no secondary generalization.

\section{Anatomical Dissections}

Three cadaveric human head specimens were prepared for the white matter dissection technique, with the specimens extracted in the first 12 hours postmortem and placed in a $10 \%$ formalin solution for 8 weeks. After this time, the heads were washed under fresh running water for a couple of hours and frozen down to $-15^{\circ} \mathrm{C}$ for 2 weeks. After this preparation, a frontally extended pterional craniotomy was bilaterally performed and the skull was elevated in one piece. White matter dissection was then performed from lateral to medial and medial to lateral on each of the six hemispheres. Each step of the dissection was photographed on the skull base and also covered by the calvaria with the previously performed frontopterional craniotomy in order to show the relationships of the involved white matter fiber tracts to the edges of the bony exposure.

The human brain develops continually from infancy to adolescence. During this period, important developmental processes occur in terms of fine motor, affective, and cognitive functions, even though sensory development is almost complete at 5-7 years. ${ }^{1}$ There are several crosssectional MRI and, more recently, diffusion tensor imaging studies showing that almost all prominent white matter tracts can be identified from birth in their well-known classic location. However, it is their anisotropy that was often low. The evolution of the fractional anisotropy, shape, and size of the white matter tracts comprised generally three phases: rapid changes during the first 12 months, 

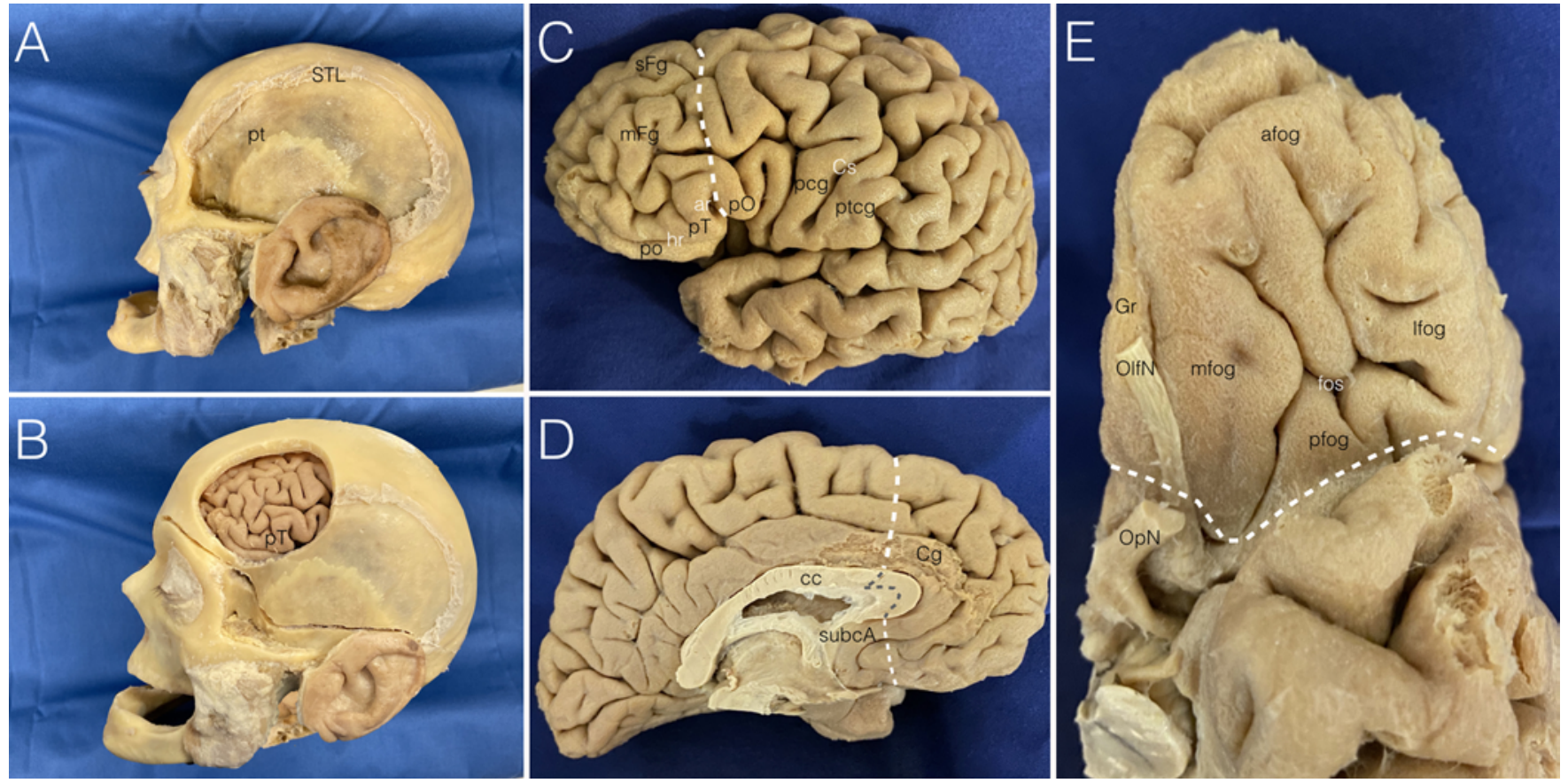

FIG. 4. Left lateral view (A) of a cadaveric specimen showing the skull and its relationship to the superior temporal line (STL) and pterion (pt). Left frontally extended pterional craniotomy and dural opening (B) showing the pars triangularis (pT), orbitals, and opercularis as well as the middle frontal gyrus. Lateral (C), medial (D), and basal $(E)$ views of a left hemisphere specimen in which the corticotomies are represented by white dashed lines. afog = anterior frontoorbital gyrus; ar = sylvian fissure ascending ramus; $\mathrm{cc}=$ corpus callosum; $\mathrm{Cg}=$ cingulate gyrus; $\mathrm{Cs}$ = central sulcus; fos = frontoorbital sulcus; $\mathrm{Gr}=$ gyrus rectus; $\mathrm{hr}=$ sylvian fissure horizontal ramus; Ifog = lateral frontoorbital gyrus; $\mathrm{mFg}=$ middle frontal gyrus; $\mathrm{mfog}=$ middle frontoorbital gyrus; OlfN = olfactory nerve; $\mathrm{OpN}$ = optic nerve; $\mathrm{pcg}$ = precentral gyrus; $p f o g=$ posterior frontoorbital gyrus; $\mathrm{po}=$ pars orbitalis; $\mathrm{pO}=$ pars opercularis; ptcg = postcentral gyrus; $\mathrm{sFg}=$ superior frontal gyrus; subcA = subcallosal area.

slow modifications during the 2nd year, and relative stability after 24 months. ${ }^{20}$

The superior frontal area is thought to be responsible for higher cortical functions such as working memory, inhibition, and attention. Thus, this white matter region would be expected to develop substantially during childhood and adolescence. Notably, as in other peripheral subcortical areas, the change in fractional anisotropy in superior frontal area was found to be greater than in many deep white matter regions..$^{30}$

Thus, we can conclude that the main anatomical subcortical net is developed as the root for subsequent "maturation" during childhood and adolescence. The direction and relative location of the white matter fibers appear to be the same. However, big changes in fractional anisotropy, shape, and size appear to be relevant during the growing periods. We cannot fully affirm that these frontal fibers are equal in adults and children; however, the 3D orientation and connecting areas appear to be very similar, or even the same.

\section{Surgical Technique}

After induction of general anesthesia, the patient is placed in the dorsal decubitus position with the head turned toward the opposite side (Video 1).

VIDEO 1. Video clip showing a unilateral prefrontal lobotomy performed in a 5-month-old boy with refractory epilepsy. The different surgical steps, namely the intrafrontal disconnection, anterior callosotomy, and frontobasal disconnection, are detailed. Copyright University Hospital of Lausanne. Published with permission. Click here to view.

A bicoronal incision is made to obtain a basal exposure, and the cutaneous flap and superior portion of the temporal muscle are elevated and retracted. A large unilateral frontal craniotomy is performed. The dura mater is opened, allowing extensive exposure of the sylvian fissure, the superior temporal gyrus, and the frontal lobe up to the precentral gyrus posteriorly (Fig. 3).

\section{Electrophysiological Monitoring}

The central sulcus, precentral gyrus, and sylvian fissure should be identified based on the sulcogyral anatomy and confirmed with electrophysiological localization.

The prefrontal lobe is the part of the frontal lobe anterior to the precentral sulcus and is composed of the superior, middle, and inferior frontal gyri on the dorsal cortical surface. Cortical dysplasia, as well as other congenital or acquired conditions, can distort the normal anatomy, and in such cases electrophysiological monitoring remains key to identifying the sulcal patterns. A quadripolar subdural platinum electrode (AD-TECH) is positioned on the cerebral cortex. The phase inversion between the frontal (P22) and the parietal (N20) response to the contralateral median nerve stimulation $(3.7 \mathrm{~Hz}, 10 \mathrm{~mA}, 200 \mu \mathrm{sec})$ indicates the position of the central sulcus. Contralateral mo- 

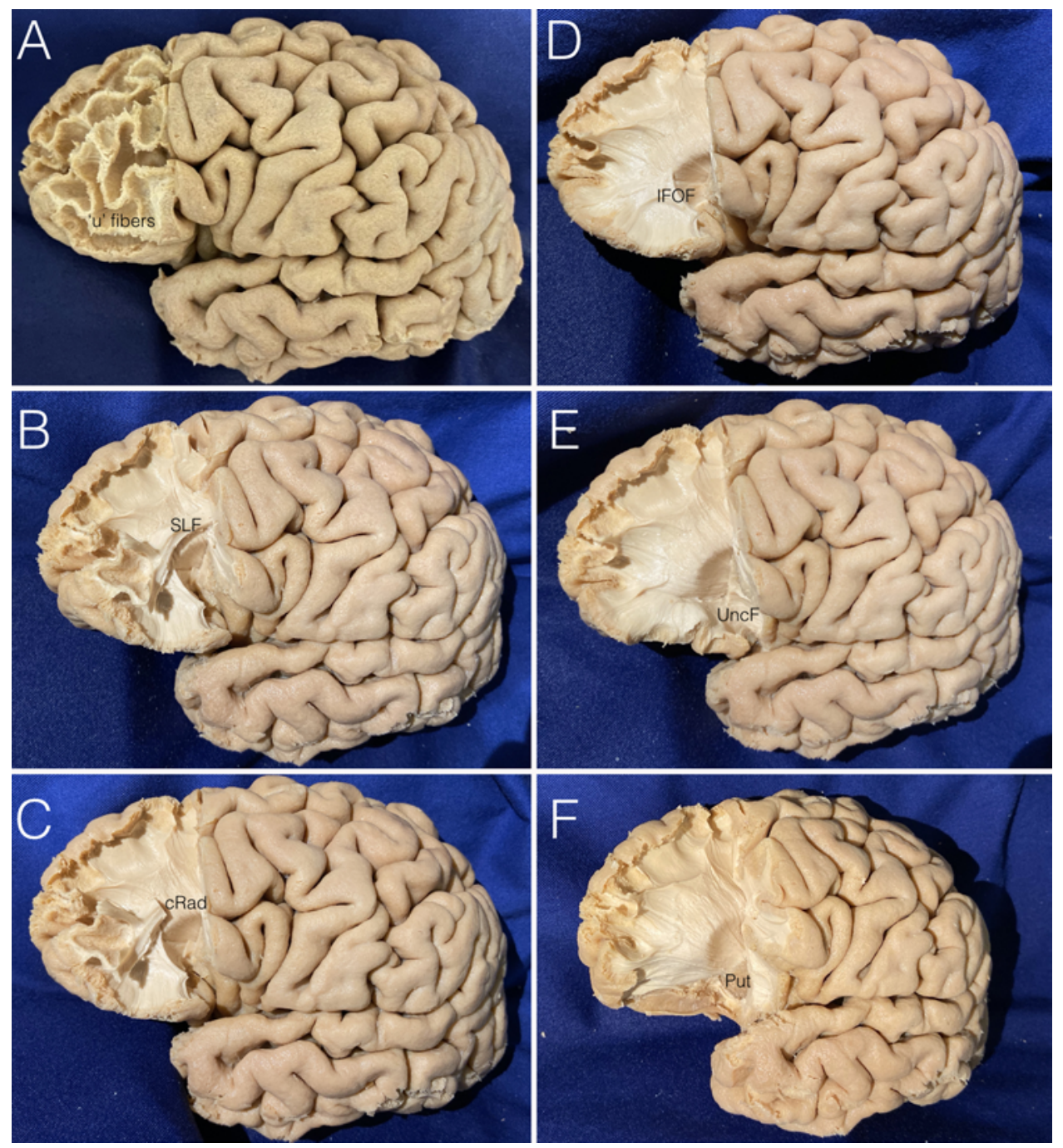

FIG. 5. Left hemisphere of a cadaveric human brain in which the lateral aspect dissection has been performed, showing the fibers disconnected during the prefrontal lobotomy procedure. After removing the cortical gray matter $(\mathbf{A})$, the first fiber tracts exposed are a group of short association, or intergyral, fibers, also known as "U-fibers" because of their shape in joining adjacent gyri. These tracts have been exposed in the left frontal lobe in between the superior, middle, and inferior frontal gyri just anterior to a vertical line traced upward from the anterior sylvian point parallel to the ascending ramus of the sylvian fissure. After removing the $\mathrm{U}$-fibers (B), the dorsal long association fiber system is exposed. This complex is represented by the rostral aspect of the superior longitudinal fascicle (SLF; anterior aspect), which connects the frontal with the central, parietal, occipital, and temporal lobes. This white matter fiber tract runs deep to the middle frontal gyrus. The next step in the dissection (C) involves cutting the inferior frontal gyrus stem to expose the insular apex as well as the short association insuloopercular fibers connecting the anterior insular lobe to the pars triangularis and opercularis (extreme capsule). Underlying these fibers, the external and internal capsules join to give rise to the corona radiata (cRad) in the superior border of the putamen. In a ventral location, the frontal and frontoorbital parts of the inferior frontooccipital fascicle (IFOF; D) and uncinate fascicle (UncF; E) are exposed. After removing the insular apex and extreme and external capsules, the lateral aspect of the putamen (Put; $\mathbf{F}$ ) is uncovered.

tor responses to cortical anodic stimulation (train-of-five, $500 \mathrm{~Hz}, 200$ usec, monopolar, $5 \mathrm{~mA}$ ) confirm localization of the motor cortex.

During the white matter tract disconnection, the monopolar stimulation is switched from anodic to cathodic stimulation, which helps to avoid damage to the motor fibers of the corona radiata. Furthermore, continuous somatosensory evoked potential monitoring is performed during the surgical procedure.

Intraoperative electrocorticography is then performed with the help of surface electrodes placed on the cortex under direct visual control to establish extensions of the epileptogenic zone.

\section{Surgical Steps \\ Intrafrontal Disconnection}

After confirmation of the epileptogenic focus situated anterior to the pars triangularis of the inferior frontal gyrus via electrocorticography, the disconnection procedure is initiated by a cortical incision placed between the pars 


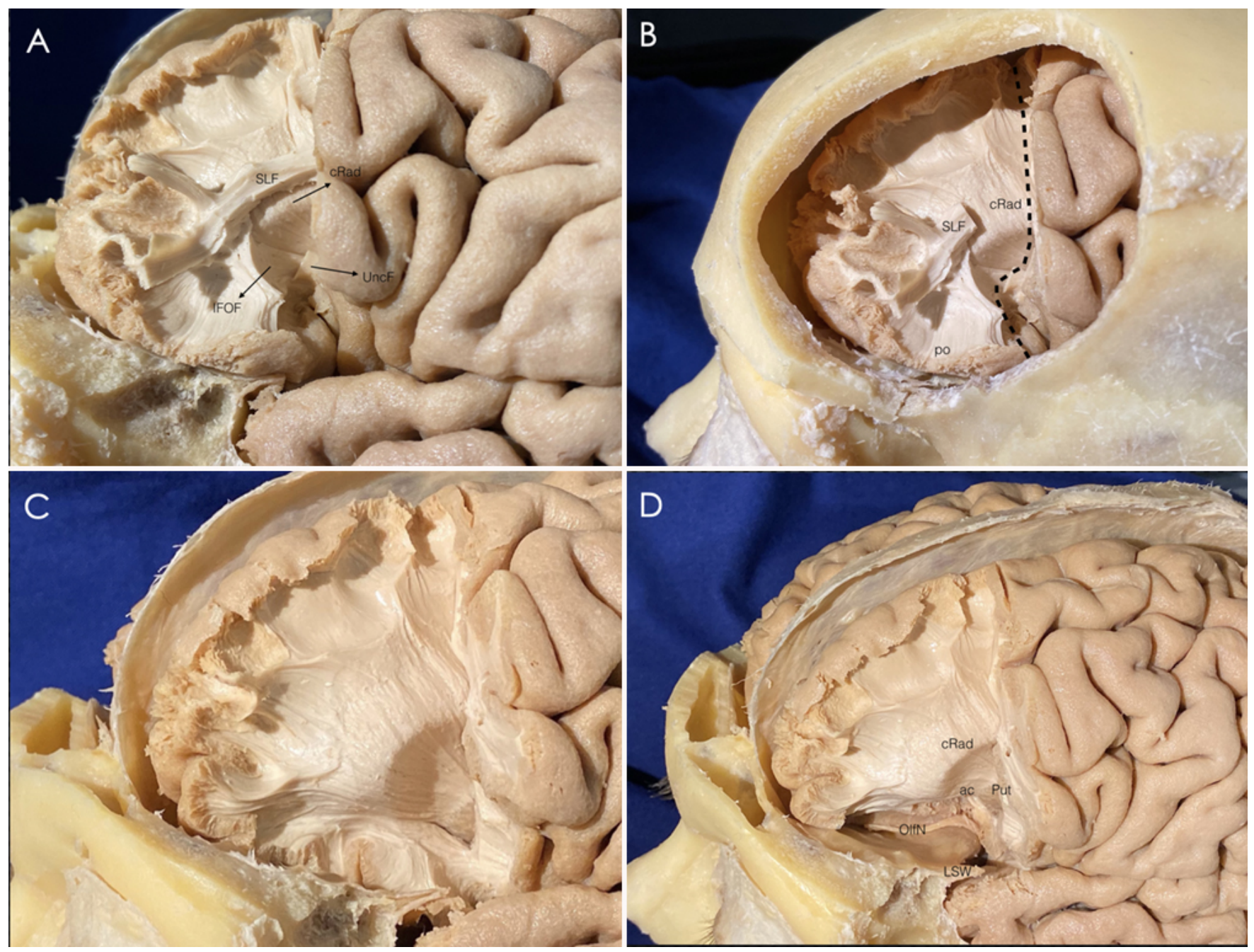

FIG. 6. Left hemisphere of a cadaveric human brain over the anterior skull base, where the main fibers in the lateral aspect are exposed. The corticotomy has been performed running upward following a parallel line to the ascending ramus of the sylvian fissure. The 3D relationships (A) between the superior longitudinal fascicle (SLF), corona radiata (CRad), inferior frontooccipital fascicle (IFOF), and uncinate fascicle (UncF) are visible. All of these frontal components of the association and projection systems are finally disconnected from remaining hemisphere during the anterior prefrontal lobotomy. The brain is visible through a frontally extended pterional craniotomy (B), illustrating the surgical disconnection plane and how this line disconnects the SLF, IFOF, and UncF, as well as the prefrontal and frontoorbital extensions of the cRad. The frontal extension of the main dorsal (superior longitudinal) and ventral (inferior frontooccipital and uncinate) association tracts has been removed (C) to expose the whole cortical extension of the anterior limb of the internal capsule (corticospinal, corticopontine, corticostriatal, and medially located thalamocortical fibers). All four frontoorbital gyri have been removed (D), showing the lateral aspect of the gyrus rectus just medial to the olfactory sulcus and olfactory nerve (OlfN). Its close relationship to the sphenoid ridge is visible. Medially, the area where the olfactory trigone, anterior commissure (ac), ventral putaminal aspect, and anterior perforated substance are exposed can be appreciated. The medial subpial resection at the frontobasal area will cross through anterior to this hot spot in order to avoid vascular complications. LSW = lesser sphenoid wing; po = pars orbitalis; Put = putamen.

opercularis and the pars triangularis (Figs. 3A, 3B, and 4) and is then continued superiorly toward the vertex and deepened in the direction of the frontal horn of the ipsilateral ventricle (Fig. 3C and D) and superiorly till the falx is reached (Fig. 3E). This disconnection line lies just anterior to a vertical line traced upward from the anterior sylvian point parallel to the ascending ramus of the sylvian fissure.

Once the cortical gray matter is removed, the first fiber tracts exposed are the U-fibers connecting the superior, middle, and inferior frontal gyri (Fig. 5A). After removing these U-fibers (Fig. 5B), at the inferior part of the disconnection between the pars opercularis and triangularis, the insular apex, as well as the short association insuloopercular fibers connecting the anterior insular lobe to the frontal stem, is cut (extreme capsule; Fig. 5C). Ventrally, the frontal and frontoorbital parts of the inferior frontooccipital fascicle (Fig. 5D) and uncinate fascicle (Fig. 5E), extending from the medial portion of the temporal lobe and the basal frontal lobe, are also cut.

The rostral portion of the superior longitudinal fascicle runs deep to the middle frontal gyrus. The connections 

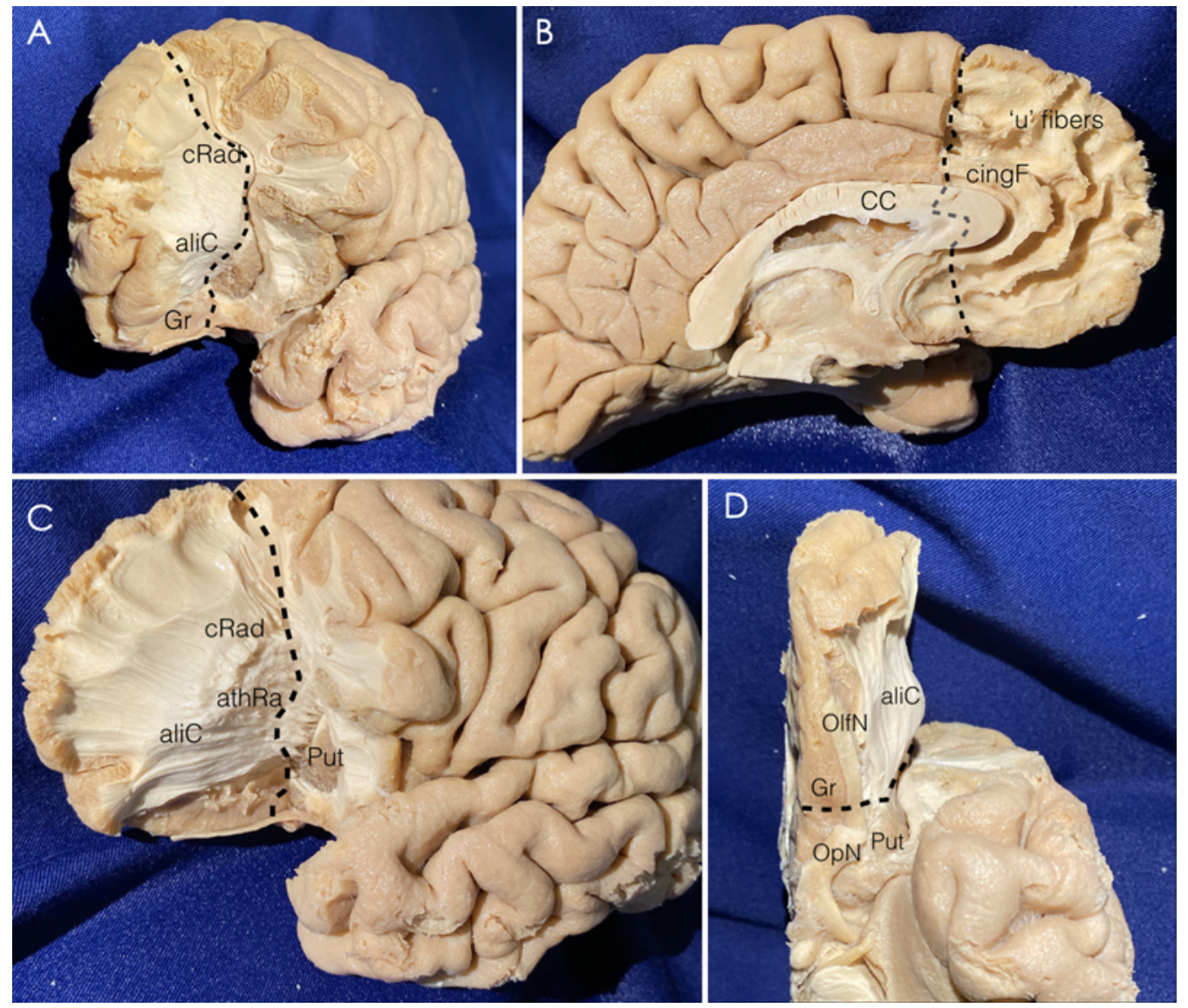

FIG. 7. Cadaveric left hemisphere, anterolateral view (A), showing the disconnection line (black dashed line) running downward from the medial aspect of the hemisphere at the superior frontal gyrus to the gyrus rectus (Gr). Left hemisphere, medial view (B), after removing the cortical aspect of the anterior half of the frontal lobe. The disconnection line (black dashed line) is shown running downward, crossing the medial frontal and cingulate fascicle (cingF). After entering the ventricle laterally, the disconnection line runs superior and anterior to the head of the caudate, also disconnecting the anterior third of the body, genu, and rostrum of the corpus callosum (CC), finally crossing the septal and subcallosal areas. From a lateral view (C), once the corticopontine fibers have been removed, a group of anteriorly directed fibers is visible. These are the anterior thalamic radiations (athRa) coming from the anterior thalamic nuclei toward the prefrontal cortex. Basal view of the same specimen (D) showing the relationship of these anteriorly directed fibers with the frontobasal disconnection line (black dashed line) crossing the $\mathrm{Gr}$ in its posterior third just a few millimeters anterior to the anterior perforated substance. aliC = anterior limb of the internal capsule; $\mathrm{CRad}=\mathrm{corona}$ radiata; OIfN = olfactory nerve; $\mathrm{OpN}=$ optic nerve; Put = putamen.

between the frontal and the central, parietal, occipital, and temporal lobes are cut at this stage (Figs. 5B and 6B). More deeply, the anterior limb of the internal capsule is then sectioned, along with the corticopontine, corticostriatal, and medially located thalamocortical fibers (Fig. 6). The 3D representation of the white matter tracts cut during the procedure is featured in Fig. 6.

\section{Anterior Callosotomy}

Once the ventricle is reached along a line superior and anterior to the head of the caudate, the disconnection is continued superiorly to cut the genu of the callosum till the pericallosal cistern and the anterior cerebral arteries are reached. The disconnection is then continued anteriorly to cut the rostrum of the corpus callosum (Fig. 3F). During this step, the forceps minor, which is a structure that connects the prefrontal lobes on either side, is sectioned (Figs. 7 and 8).

\section{Frontobasal Disconnection}

From the surface of the inferior frontal gyrus, the cut is deepened to disconnect the basal and inferomesial portions of the frontal lobe (Fig. 4D and E). The section follows the direction of the sphenoid ridge and is performed through the basal white matter of the orbital and straight gyri, while respecting the basal pia (Fig. 6D). While performing the subpial disconnection, the posteromedial part represents a crucial component at which the disconnection should remain subpial. The olfactory tract and contents of the carotid and proximal sylvian cisterns seen through the pia can help guide the disconnection in its entirety. When the medial surface of the hemisphere is reached, the cut joins the section of the rostrum and allows visualization of the carotid bifurcation in transparency across the pia and arachnoidal planes.

During this phase the anterior limb of the anterior commissure is sectioned, thus limiting the afferences from the 

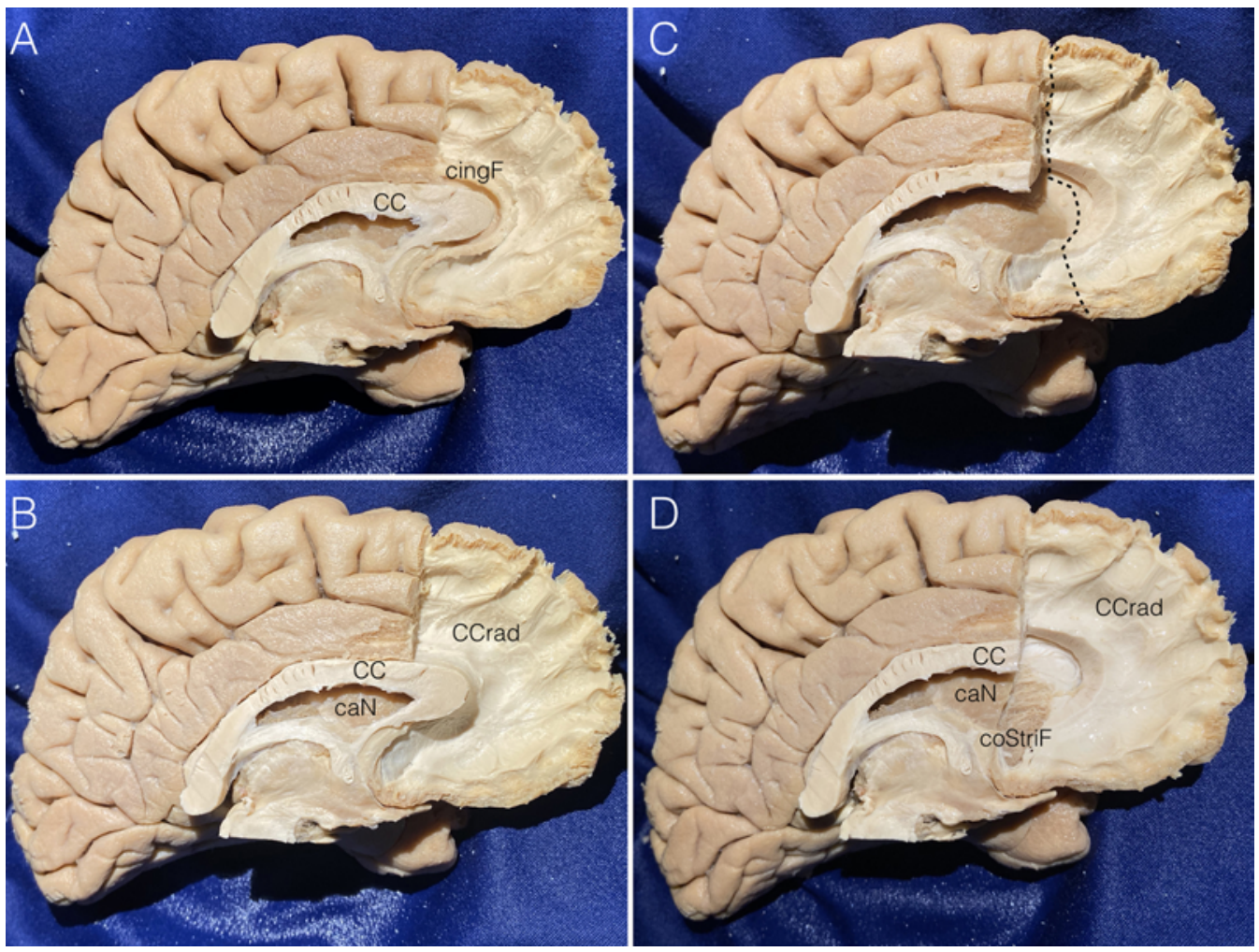

FIG. 8. Consecutive steps of the dissection from the medial view of the left hemisphere. The anterior third of the cingulate fascicle (cingF) is exposed after removing the cortical layer of the medial frontal lobe (A). Once the cing $F$ has been removed, the whole medial extension of the anterior third of the corpus callosum (CC) is clearly visible (B). A dotted black line (C) represents the subpial disconnection in the medial aspect of the hemisphere. This maneuver disconnects the anterior projections of the corona radiata, as well as the cingF, anterior third of the body, genu, and rostrum of the $\mathrm{CC}$, as well as the frontobasal projections and the thin commissural fibers of the anterior commissure joining the olfactory areas. After removing the gray matter of the head of the caudate nucleus (caN; D), a medial view of the corticostriatal fibers (coStriF) is visible in the depth of the caudate. CCrad = corpus callosum frontal radiations.

cingulum and the limbic system (paraterminal and paraolfactory areas; Fig. 7C and D).

\section{Discussion}

Frontal lobe resections account for a third of the lobar resections in pediatric epilepsy surgery. ${ }^{19}$ The diagnosis and surgical treatment of FLE are often significantly more complex than those of temporal lobe epilepsy. Seizure semiology is the key to differentiating types of FLE.,32 The prefrontal cortex is involved in emotion processing, moral behavior, executive functions, working memory, and learning, and seizures typically arising from this area are hypermotor seizures, which are associated with the preservation of consciousness, usually occur during sleep, and are preceded by an aura. ${ }^{3}$ Their complex semiology may be caused by the disruption of neuronal synchrony between different cerebral regions. ${ }^{2}$

Here, we report a disconnective procedure of the prefrontal lobe (unilateral prefrontal lobotomy) as a surgical alternative to resective procedures to treat large epileptogenic areas in the frontal lobe.

In FLE patients, the probability of becoming seizure free is $55.7 \%$ at 1 year, $45 \%$ at 3 years, and $30 \%$ at 5 years. ${ }^{21}$ However, pediatric series have reported better outcomes than those in adult series, with seizure arrest in more than $60 \%$ of cases. ${ }^{25,34,41}$ Englot et al. ${ }^{17}$ performed a meta-analysis on the subject and reported more favorable seizure outcomes among individuals with tumors, cortical dysplasia, or other focal lesions. Higher rates of seizure freedom have been noted after partial frontal lobectomy involving the anterior/polar, dorsolateral, mesial, or convexity regions, as compared to the rates following more extensive frontal lobectomies or resections. Gross-total resection in lesional FLE has been associated with a significantly higher rate of seizure freedom $(60.8 \%)$ than that with subtotal lesionectomy (36.4\%). ${ }^{17}$ Focal cortical dysplasia is one of the most common histopathological findings causing extratemporal refractory epilepsy in children and adolescents and is associated with a better surgical outcome than those with other etiologies., ${ }^{419,34}$ The radiological diagnosis of focal cortical dysplasia can be challenging, ${ }^{31}$ and the correct definition of its limits is essential to perform a complete lesionectomy or disconnection of the epileptogenic zone.

Unclear MRI lesions, diffuse ictal onset on EEG, and incomplete removal of the epileptogenic zone remain negative prognostic factors. ${ }^{17,21}$ Factors associated with a better outcome in FLE include abnormal MRI findings (well defined), lateralized ictal scalp EEG pattern, short 
duration of epilepsy, younger age at surgery, and circumscribed histopathological findings.,3,17,29 A greater distance between the epileptogenic zone and eloquent areas has also been described as a favorable prognostic factor. ${ }^{14}$ In pediatric patients, there is increasing evidence that a shorter duration of epilepsy is associated with a better surgical cure..$^{33,34,38}$ Pediatric epilepsy surgery studies have stressed the importance of early surgery to address both seizure outcomes and developmental delay. ${ }^{23,28,34}$ A younger age at surgery may also allow limitation of postoperative deficits as they can be overcome by more important plasticity mechanisms. ${ }^{34}$ For principally these reasons, we decided to perform the surgery in our case as soon as it was deemed safe from an anesthesia standpoint.

The risk of potential deficits and adverse effects must be carefully weighed against surgical benefit in planning surgery for FLE. Intraoperative electrophysiological mapping and monitoring are invaluable for these interventions. Subdural grids and depth electrodes may help in better defining the epileptogenic zone. The use of motor tract electrophysiological monitoring is of the outmost importance for surgery in lesions adjacent to motor and insular areas and helps in limiting postoperative deficits.

For disconnective epilepsy surgery in general, the key to safe disconnection is the ability to remain in the subpial plane. Therefore, all stages of the cortical disconnection (except over the convexity) should be performed in this plane, which ensures the preservation of vessels, especially the perforating arteries. Visualization of the cisternal structures through the pia can very often guide the disconnection procedure at these stages of the surgery.

Disconnective surgery has been well accepted in hemispheric and subhemispheric epilepsy syndromes. There have been several studies involving a host of techniques for hemispheric diseases, namely variants of lateral and vertical hemispherotomies, which all attest to low perioperative morbidity, possibly due to decrements in intraoperative blood loss and surgical duration..$^{13,37,42}$ In addition, the rates of hydrocephalus are also considerably less in the modern era of hemispheric epilepsy surgery in which the majority of epilepsy surgery centers use disconnective techniques. ${ }^{12,37}$ There has been a similar trend for subhemispheric epilepsy, for which there have been several recent reports on the use of disconnective techniques in anterior and posterior quadrantic epilepsy syndromes, demonstrating their effectiveness while maintaining low rates of complications. ${ }^{6,7,9-11}$ The value of disconnections over resections for large epileptogenic lesions is of particular importance in the pediatric population given that these syndromes are almost always treated in this particular age group. However, the benefits of disconnections over resection in lobar pathologies remain to be proved.

\section{Conclusions}

Unilateral prefrontal lobotomy represents an alternative technique to resection in the treatment of extensive cortical dysplasia involving the frontal lobe unilaterally. Long-term seizure control for these lesions is dependent on the presence of a well-defined electroencephalographic epileptogenic zone distant from eloquent areas. The ad- vantages of disconnection over resection could have particular value in infants because of its benefits in reducing perioperative morbidity, while its value in terms of seizure control needs to be assessed in larger patient cohorts.

\section{References}

1. Ashtari M, Cervellione KL, Hasan KM, Wu J, McIlree C, Kester $\mathrm{H}$, et al: White matter development during late adolescence in healthy males: a cross-sectional diffusion tensor imaging study. Neuroimage 35:501-510, 2007

2. Bartolomei F, Trébuchon A, Gavaret M, Régis J, Wendling F, Chauvel P: Acute alteration of emotional behaviour in epileptic seizures is related to transient desynchrony in emotionregulation networks. Clin Neurophysiol 116:2473-2479, 2005

3. Beleza P, Pinho J: Frontal lobe epilepsy. J Clin Neurosci 18:593-600, 2011

4. Blumcke I, Russo GL, Najm I, Palmini A: Pathology-based approach to epilepsy surgery. Acta Neuropathol 128:1-3, 2014

5. Chang EF, Wang DD, Barkovich AJ, Tihan T, Auguste KI, Sullivan JE, et al: Predictors of seizure freedom after surgery for malformations of cortical development. Ann Neurol 70:151-162, 2011

6. Cossu G, Lebon S, Seeck M, Pralong E, Messerer M, RouletPerez E, et al: Periinsular anterior quadrantotomy: technical note. J Neurosurg Pediatr 21:124-132, 2018

7. D'Agostino MD, Bastos A, Piras C, Bernasconi A, Grisar T, Tsur VG, et al: Posterior quadrantic dysplasia or hemihemimegalencephaly: a characteristic brain malformation. Neurology 62:2214-2220, 2004

8. D’Argenzio L, Colonnelli MC, Harrison S, Jacques TS, Harkness W, Vargha-Khadem F, et al: Cognitive outcome after extratemporal epilepsy surgery in childhood. Epilepsia 52:1966-1972, 2011

9. Daniel RT, Babu KS, Jacob R, Villemure J: Posterior quadrantic resection and disconnection, in Cataltepe O, Jallo GI (eds): Pediatric Epilepsy Surgery: Preoperative Assessment and Surgical Treatment. New York: Thieme Medical Publisher, 2010, pp 196-204

10. Daniel RT, Meagher-Villemure K, Farmer JP, Andermann F, Villemure JG: Posterior quadrantic epilepsy surgery: technical variants, surgical anatomy, and case series. Epilepsia 48:1429-1437, 2007

11. Daniel RT, Meagher-Villemure K, Roulet E, Villemure JG: Surgical treatment of temporoparietooccipital cortical dysplasia in infants: report of two cases. Epilepsia 45:872-876, 2004

12. Daniel RT, Villemure JG: Peri-insular hemispherotomy: potential pitfalls and avoidance of complications. Stereotact Funct Neurosurg 80:22-27, 2003

13. Delalande O, Bulteau C, Dellatolas G, Fohlen M, Jalin C, Buret V, et al: Vertical parasagittal hemispherotomy: surgical procedures and clinical long-term outcomes in a population of 83 children. Neurosurgery 60 (2 Suppl 1):ONS19ONS32, 2007

14. Delev D, Oehl B, Steinhoff BJ, Nakagawa J, Scheiwe C, Schulze-Bonhage A, et al: Surgical treatment of extratemporal epilepsy: results and prognostic factors. Neurosurgery 84:242-252, 2019

15. Dorfer C, Czech T, Mühlebner-Fahrngruber A, Mert A, Gröppel G, Novak K, et al: Disconnective surgery in posterior quadrantic epilepsy: experience in a consecutive series of 10 patients. Neurosurg Focus 34(6):E10, 2013

16. Elsharkawy AE, Alabbasi AH, Pannek H, Schulz R, Hoppe $\mathrm{M}$, Pahs G, et al: Outcome of frontal lobe epilepsy surgery in adults. Epilepsy Res 81:97-106, 2008

17. Englot DJ, Wang DD, Rolston JD, Shih TT, Chang EF: Rates 
and predictors of long-term seizure freedom after frontal lobe epilepsy surgery: a systematic review and meta-analysis. J Neurosurg 116:1042-1048, 2012

18. Fish DR, Smith SJ, Quesney LF, Andermann F, Rasmussen T: Surgical treatment of children with medically intractable frontal or temporal lobe epilepsy: results and highlights of 40 years' experience. Epilepsia 34:244-247, 1993

19. Harvey AS, Cross JH, Shinnar S, Mathern GW: Defining the spectrum of international practice in pediatric epilepsy surgery patients. Epilepsia 49:146-155, 2008 (Erratum in Epilepsia 54:1140, 2013)

20. Hermoye L, Saint-Martin C, Cosnard G, Lee SK, Kim J, Nassogne MC, et al: Pediatric diffusion tensor imaging: normal database and observation of the white matter maturation in early childhood. Neuroimage 29:493-504, 2006

21. Jeha LE, Najm I, Bingaman W, Dinner D, Widdess-Walsh P, Lüders H: Surgical outcome and prognostic factors of frontal lobe epilepsy surgery. Brain 130:574-584, 2007

22. Jehi L, Sarkis R, Bingaman W, Kotagal P, Najm I: When is a postoperative seizure equivalent to "epilepsy recurrence" after epilepsy surgery? Epilepsia 51:994-1003, 2010

23. Jonas R, Asarnow RF, LoPresti C, Yudovin S, Koh S, Wu JY, et al: Surgery for symptomatic infant-onset epileptic encephalopathy with and without infantile spasms. Neurology 64:746-750, 2005

24. Kazemi NJ, So EL, Mosewich RK, O'Brien TJ, Cascino GD, Trenerry MR, et al: Resection of frontal encephalomalacias for intractable epilepsy: outcome and prognostic factors. Epilepsia 38:670-677, 1997

25. Kral T, Kuczaty S, Blümcke I, Urbach H, Clusmann H, Wiestler OD, et al: Postsurgical outcome of children and adolescents with medically refractory frontal lobe epilepsies. Childs Nerv Syst 17:595-601, 2001

26. Lazow SP, Thadani VM, Gilbert KL, Morse RP, Bujarski KA, Kulandaivel K, et al: Outcome of frontal lobe epilepsy surgery. Epilepsia 53:1746-1755, 2012

27. Lee JJ, Lee SK, Lee SY, Park KI, Kim DW, Lee DS, et al: Frontal lobe epilepsy: clinical characteristics, surgical outcomes and diagnostic modalities. Seizure 17:514-523, 2008

28. Loddenkemper T, Holland KD, Stanford LD, Kotagal P, Bingaman W, Wyllie E: Developmental outcome after epilepsy surgery in infancy. Pediatrics 119:930-935, 2007

29. McIntosh AM, Averill CA, Kalnins RM, Mitchell LA, Fabinyi GC, Jackson GD, et al: Long-term seizure outcome and risk factors for recurrence after extratemporal epilepsy surgery. Epilepsia 53:970-978, 2012

30. Moon WJ, Provenzale JM, Sarikaya B, Ihn YK, Morlese J, Chen S, et al: Diffusion-tensor imaging assessment of white matter maturation in childhood and adolescence. AJR Am J Roentgenol 197:704-712, 2011

31. Noe K, Sulc V, Wong-Kisiel L, Wirrell E, Van Gompel JJ, Wetjen N, et al: Long-term outcomes after nonlesional extratemporal lobe epilepsy surgery. JAMA Neurol 70:10031008,2013

32. Ostergard TA, Miller JP: Surgery for epilepsy in the primary motor cortex: a critical review. Epilepsy Behav 91:13-19, 2019

33. Pinheiro-Martins AP, Bianchin MM, Velasco TR, Terra VC, Araújo D Jr, Wichert-Ana L, et al: Independent predictors and a prognostic model for surgical outcome in refractory frontal lobe epilepsy. Epilepsy Res 99:55-63, 2012

34. Ramantani G, Kadish NE, Mayer H, Anastasopoulos C, Wagner K, Reuner G, et al: Frontal lobe epilepsy surgery in childhood and adolescence: predictors of long-term seizure freedom, overall cognitive and adaptive functioning. Neurosurgery 83:93-103, 2018
35. Rasmussen T: Tailoring of cortical excisions for frontal lobe epilepsy. Can J Neurol Sci 18 (4 Suppl):606-610, 1991

36. Schramm J, Kral T, Kurthen M, Blümcke I: Surgery to treat focal frontal lobe epilepsy in adults. Neurosurgery 51:644654,2002

37. Schramm J, Kuczaty S, Sassen R, Elger CE, von Lehe M: Pediatric functional hemispherectomy: outcome in 92 patients. Acta Neurochir (Wien) 154:2017-2028, 2012

38. Simasathien T, Vadera S, Najm I, Gupta A, Bingaman W, Jehi L: Improved outcomes with earlier surgery for intractable frontal lobe epilepsy. Ann Neurol 73:646-654, 2013

39. Téllez-Zenteno JF, Dhar R, Wiebe S: Long-term seizure outcomes following epilepsy surgery: a systematic review and meta-analysis. Brain 128:1188-1198, 2005

40. Thomas SG, Chacko AG, Thomas MM, Babu KS, Russell PS, Daniel RT: Outcomes of disconnective surgery in intractable pediatric hemispheric and subhemispheric epilepsy. Int J Pediatr 2012:527891, 2012

41. Vachhrajani S, de Ribaupierre S, Otsubo H, Ochi A, Weiss SK, Donner EJ, et al: Neurosurgical management of frontal lobe epilepsy in children. J Neurosurg Pediatr 10:206-216, 2012 (Erratum in J Neurosurg Pediatr 10:565, 2012)

42. Villemure JG, Mascott CR: Peri-insular hemispherotomy: surgical principles and anatomy. Neurosurgery 37:975-981, 1995

43. Yun CH, Lee SK, Lee SY, Kim KK, Jeong SW, Chung CK: Prognostic factors in neocortical epilepsy surgery: multivariate analysis. Epilepsia 47:574-579, 2006

44. Zaatreh MM, Spencer DD, Thompson JL, Blumenfeld H, Novotny EJ, Mattson RH, et al: Frontal lobe tumoral epilepsy: clinical, neurophysiologic features and predictors of surgical outcome. Epilepsia 43:727-733, 2002

45. Zentner J, Hufnagel A, Ostertun B, Wolf HK, Behrens E, Campos MG, et al: Surgical treatment of extratemporal epilepsy: clinical, radiologic, and histopathologic findings in 60 patients. Epilepsia 37:1072-1080, 1996

\section{Disclosures}

The authors report no conflict of interest concerning the materials or methods used in this study or the findings specified in this paper.

\section{Author Contributions}

Conception and design: Daniel, Cossu. Acquisition of data: Daniel, Cossu, González-López. Analysis and interpretation of data: Cossu, González-López, Pralong, Kalser. Drafting the article: Daniel, Cossu, González-López, Kalser, Messerer. Critically revising the article: Daniel, Cossu, Pralong, Messerer. Reviewed submitted version of manuscript: all authors. Approved the final version of the manuscript on behalf of all authors: Daniel. Administrative/technical/material support: Cossu, Pralong. Study supervision: Daniel, Messerer.

\section{Supplemental Information \\ Videos}

Video 1. https://vimeo.com/390966951.

\section{Correspondence}

Roy Thomas Daniel: University Hospital of Lausanne, Switzerland.roy.daniel@chuv.ch. 